\title{
Análisis de Procesos Simultáneos Reacción - Extracción a Mivel Productivo. Generalidades del Proceso, Equilibrios Fásico y Químico Simultáneos
}

\author{
Marcela Rivera* \\ Carlos Ariel Cardona**
}

* Ingeniera Química - Universidad Macional de Colombia, Manizales, Colombia

e-mail:marcelariveravillegas@yahoo.com

** Ph.D. Profesor Asistente - Universidad Macional de Colombia, Manizales, Colombia.

e-mail:ccardona@nevado.manizales.unal.edu.co

Fecha de recepción: Marzo 9 de 2004

Fecha de aprobación: Septiembre 15 de 2004

\section{RESUMEN}

Se presentan los fundamentos teóricos de los procesos simultáneos reacción extracción. Se analiza el equilibrio químico y de fases simultáneo para mezclas multicomponentes, con representaciones gráficas para tres y cuatro componentes.

Estos diagramas son la base del análisis termodinámico topológico que se aplica en el diseño preliminar de los procesos de reacción y fermentación extractiva, que se explica en detalle en la parte dos de este artículo. 
Palabras Claves: Reacción / Fermentación Extractiva, equilibrio químico y fásico simultáneo, recuperación de producto in situ.

\begin{abstract}
The theoretical principles of the simultaneous reaction-separation processes are presented. The simultaneous chemical and phase equilibria for multi-component mixtures are analysed, with presentation of phase diagrams for three and/or four components. These diagrams are the basis for the thermodynamic-topological analysis applied in the preliminary design of the extractive reaction/fermentation processes. It is developed in more detail in part two of this article.
\end{abstract}

Key Words: Extractive Reaction/Fermentation, simultaneous chemical and phase equilibrium, in situ product recovery (ISPR).

\section{INTRODUCCIÓN}

Con el auge en la implementación e investigación de procesos simultáneos, entre ellos la Destilación Reactiva [1-3] se genera la inquietud de extender los métodos de análisis desarrollados para esta técnica a otros procesos como son los de Reacción Extracción.

Entre estos procesos se encuentran la Extracción Reactiva, ampliamente estudiada a nivel teórico [4] y experimental [5-7], la Fermentación Extractiva $[8,9]$ y la Reacción Extractiva $[10,11]$, objeto principal de este documento.

El análisis de la representación gráfica del equilibrio simultáneo y de los flujos del proceso, es la base para desarrollar un método corto que pueda aplicarse en la síntesis del esquema tecnológico y en la evaluación de viabilidad de dichos procesos.

\section{PROCESOS SIMULTÁNEOS}

Recientemente se ha incrementado el desarrollo de procesos simultáneos separación reacción como alternativa a los procesos convencionales, tomando como herramienta la modificación efectiva de las posibilidades termodinámicas que posee el sistema. La reacción puede ser combinada con técnicas de separación como destilación, cristalización, extracción, adsorción y separación por membranas, para conseguir ciertas ventajas, como reducir la inversión capital - sobrepasar las limitaciones del equilibrio impuestas por las reacciones reversibles, lo que no se puede conseguir con los procesos convencionales.

El mecanismo especial de interacción entre la reacción química y la transferencia de masa en un aparato, asegura la optimización de la conversión y/o selectividad, criterios clave para la reducción de costos de operación. Los procesos simultáneos no sólo se limitan a procesos Separación Reacción, y la realización simultanea de estos ya sea en un aparato o en un "complejo tecnológico" (sistema de equipos conectados por reflujos, para la realización de una tarea específica) tiene el propósito de lograr una mutua intensificación, aumento de sus posibilidades y la compacidad del esquema tecnológico [1].

Estos procesos se clasifican en Homogéneos cuando se trata de procesos físicamente similares que combinan dos o más operaciones unitarias o dos o más reacciones químicas de diferente naturaleza; o Heterogéneos si combinan simultáneamente la realización de una operación unitaria y una reacción química. En estos, la componente de transferencia de masa o el avance y selectividad de la reacción es afectada (en forma positiva o negativa) por la componente de reacción química o viceversa $[1,2]$. Conjugados en el caso de que los procesos se den en diferentes aparatos $y$ condiciones, y tan sólo el esquema tecnológico se cierre con flujos y reflujos. Simultáneos si se dan en un mismo equipo. En los últimos las componentes termodinámicas de los procesos se ven afectadas entre sí, y muchas veces se logra una redistribución de flujos en el simplex

VOLUMEN 6 - No. 1 - Septiembre de 2004 
concentracional (para procesos de separación) o una sinergia energética (para reacciones).

\begin{tabular}{|l|l|}
\hline \multicolumn{1}{|c|}{ PROCESO } & \multicolumn{1}{c|}{ TIPO } \\
\hline Reacción Extractiva. Destilación Reactiva & Heterogéneo Simultaneo \\
\hline Destilación Extractiva & Homogéneo Simultaneo \\
\hline Destilacion- Cristalizacion. Deshidratación oxidativa & Homogéneo Con jugado \\
\hline $\begin{array}{l}\text { Fermentaci ón-Extracción en la Producci on de ácido } \\
\text { butírico [9] }\end{array}$ & Heterogéneo conjuga do \\
\hline
\end{tabular}

\subsection{Análisis de la Estática:}

Entre los procesos simultáneos, los de Destilación Reactiva son los más investigados y aplicados en la industria química debido a la posibilidad de incrementar la velocidad de la reacción o de la separación y a las ventajas que ofrece en el desarrollo de reacciones reversibles. El método de diseño basado en el análisis de la termodinámica topológica fue desarrollado por Serafimov y Zharov basándose en los diagramas de Schreinemakers [12].

Más adelante se desarrolla el análisis de la estática que permite seleccionar los estados estables límites (que corresponden al máximo rendimiento del producto deseado), el diseño del proceso y su viabilidad, con la ventaja de requerir poca información (parámetros de los modelos de equilibrios de fase y químico y la estequiometría de la reacción), reducir el tiempo de cómputo y simplificar los experimentos. En las referencias [1-3, 13-15] se presenta de manera detallada la metodología del análisis de la estática.

A partir de estas investigaciones y de la concepción de Pisarenko et al.[1] de que el análisis de la estática y los métodos cortos desarrollados para Destilación Reactiva pueden ser extendidos a diversos procesos Reacción Separación, se plantea el objetivo principal de este trabajo.

\section{PROCESOS SIMULTÁNEOS REACCIÓN EXTRACCIÓN}

Los procesos simultáneos Reacción-Extracción, involucran simultáneamente y en una misma unidad, la reacción y la separación de fases Líquido - Líquido.
La inmiscibilidad de las fases líquidas se puede dar naturalmente dentro del sistema de reacción (p.e. por la formación de un producto parcialmente miscible con los reactivos) o la segunda fase puede ser introducida con la adición de solventes], logrando la separación selectiva de compuestos intermedios o productos, previniendo su posterior reacción o la inhibición de biomasa en sistemas biológicos, para dar como resultado un mayor rendimiento [10].

Estos procesos pueden ser utilizados efectivamente para aumentar de manera significativa el rendimiento, la selectividad a productos deseados y la facilidad de separación de productos secundarios, y como se mencionó anteriormente, en la mayoría de los casos, ofrecen la ventaja de reducir el número de equipos de proceso, combinando las operaciones de reacción y extracción en una sola unidad. Además representa ventajas adicionales como: disminución en los costos de la separación de productos y de tratamiento del agua de desecho, y la disminución del flujo de reciclo de reactivos no convertidos debido al incremento del rendimiento por paso. En procesos biológicos, permite la fermentación de mezclas más concentradas en sustrato.

\subsection{Tipos de Procesos de Reacción Extracción:}

$\checkmark$ Químico: En esta clase se agrupan los procesos simultáneos reacción-extracción que se aplican en la producción y/o separación de sustancias químicas por vía sintética.

Reacción Extractiva: Su principal objetivo es la síntesis de productos químicos, es decir, implementar la componente de reacción y adicionar la componente de separación para lograr la extracción selectiva de compuestos intermedios o productos para prevenir su reacción posterior y alcanzar así un mayor rendimiento. 
Esta separación in-situ conlleva a una reconcentración de reactivos con lo cual se engaña el equilibrio químico (cuando se tienen reacciones reversibles) llegando a conversiones más elevadas.

Además del efecto sinérgico alcanzado por la combinación de reacción y separación, se tiene la gran ventaja de llevar a cabo el proceso en un solo equipo.

Extracción Reactiva: Son los procesos que tienen como objetivo primario la separación. Utiliza una reacción reversible para convertir sustancias difíciles de separar, en otras sustancias cuyas características fisicoquímicas las hacen fáciles de separar.

En esta categoría podemos mencionar:

Separación o Purificación

Enriquecimiento

Conversión de sales

Extracción Reactiva Adsortiva

$\square$ Biotecnológico: Además de las aplicaciones en síntesis de productos químicos, los procesos Reacción Extracción, se ha venido implementando de manera exitosa en el campo biotecnológico, en el cual se agrupan procesos de:

Fermentación extractiva: Este proceso combina biorreacción aerobia o anaerobia con microorganismos y extracción líquido líquido in situ de los productos principales o inhibidores. Su principal ventaja es el control de la inhibición por producto final, lo cual lleva a mayores rendimientos y a la posibilidad de fermentar mezclas más concentradas en sustrato.

Reacción enzimática extractiva: Conjuga de manera simultánea las componentes de separación por extracción líquido - líquido y la reacción biológica empleando enzimas como catalizador.

\subsection{Aplicación de los Procesos de Reacción - Extracción:}

\begin{tabular}{||l|l||}
\hline \multicolumn{1}{|c|}{ PROCESO CLASIFICACION } \\
\hline $\begin{array}{l}\text { Producción de Acetato de Butilo por Ester ificación de } \\
\text { Ácido Acético }\end{array}$ & Reacción Extractiva [11] \\
\hline Separación del 1,3-propanodiol & $\begin{array}{l}\text { Extracción Reactiva } \\
\text { Purificación [7] }\end{array}$ \\
\hline $\begin{array}{l}\text { Recuperación de Penicilina G/ Ácido Láctico de caldos } \\
\text { fermentativos }\end{array}$ & $\begin{array}{l}\text { Extracción Reactiva -Adsortiva } \\
{[4]}\end{array}$ \\
\hline Producción de Etanol & Fermentación Extractiva [9] \\
\hline Producción de Ácidos Carboxílicos & Fermentación Extractiva [4] \\
\hline
\end{tabular}

\section{EQUILIBRIO LÍQUIDO - LÍQUIDO REACTIVO}

El equilibrio líquido-líquido reactivo ELLR se define para sistemas en los cuales se presente reacción química e inmiscibilidad de fases. ELLR es el estado del sistema en el cual se presenta simultáneamente equilibrio de fases y equilibrio de reacción (en adelante, para simplificar, este estado lo denominaremos equilibrio simultáneo), es decir, las fases extracto y refinado se encuentran tanto en el sub-espacio de equilibrio químico como en el de equilibrio líquido-líquido.

Considerando un sistema de $c$ componentes y $r$ reacciones químicas independientes, en el estado en el cual se presenta ELLR, se presenta equilibrio térmico y mecánico, se satisfacen simultáneamente $r$ ecuaciones de equilibrio químico (ec 2), c ecuaciones de equilibrio de fase (ec 3), tres restricciones estequiométricas (sumatorias de las fracciones molares para cada corriente) y $c$ balances de materia.

Las composiciones molares del sistema se grafican en un simplex concentracional definido como el espacio delimitado por un poliedro de $c$ lados, donde cada vértice representa un componente puro y los puntos interiores a éste representan mezclas de dichos componentes.

La separación en dos fases, se da sólo para mezclas iniciales pertenecientes a un espacio definido del simplex concentracional (región de dos fases) que se puede identificar mediante la representación gráfica del ELLR. Las mezclas 
iniciales que se encuentren por fuera de este espacio llegan al ELLR presentando una sola fase con composición dada por la composición de equilibrio químico correspondiente a dicha mezcla inicial.

\section{$\square$ Algoritmo:}

1. Informe de entrada:

Temperatura

$\mathrm{v}_{i}^{T}$ vector fila de los coeficientes estequimétricos $v$ (con signo negativo para los reactivos)

Uтот $^{T}$ Vector fila de la suma de los coeficientes estequiométricos de cada reacción.

$$
V=\left(\begin{array}{ccc}
\mathrm{U}_{(c-r+l), 1} & \ldots & \mathrm{U}_{(c-r+l), r} \\
\ldots & \mathrm{U}_{i, r} & \ldots \\
\mathbf{U}_{c, l} & \ldots & \mathrm{U}_{c, r}
\end{array}\right)
$$

$Z_{i}$ : fracciones molares iniciales de las $c$ especies

$F$ : flujo molar de mezcla inicial

$K_{m}$ : constante de equilibrio de reacción.

$m=1, \ldots, r$ (número de reacciones).

Parámetros del modelo de actividad

2 . Asumir un estimado inicial de $x_{i}$ para $i=1,2, \ldots, c$ -

$1 y_{e}$ para $\mathrm{e}=\mathrm{c}-\mathrm{r}+1 \ldots \mathrm{c}$. Y $\phi=\mathrm{R} / \mathrm{F}$ ( $x$ e $y$ son vectores columna de las composiciones molares en las fases refinado y extracto respectivamente. $\mathrm{R}$ es el flujo molar de refinado).

3. Calcular las demás $y_{i}$ a partir de los balances de materia según la ecuación:

$$
\begin{aligned}
& \mathrm{y}_{i}=\frac{\mathrm{Z}_{i}-\phi x_{i}+\mathrm{v}_{i}^{T} V^{-1}\left[\left((1-\phi) y_{e}+\phi x_{e}-z_{e}\right) /\left(1-\mathrm{v}_{T}^{T O T} \mathrm{~V}^{-1} y_{e}\right)\right]}{1-\phi+\mathrm{v}_{T}^{T O T} V^{-1}\left[\left((1-\phi) y_{e}+\phi x_{e}-z_{e}\right) /\left(1-\mathrm{U}_{T}^{T O T} \mathrm{~V}^{-1} y_{e}\right)\right]} \\
& i=1 \ldots c-r
\end{aligned}
$$

4. Calcular los coeficientes de actividad

$$
\gamma \mathrm{i}^{R}=f\left(x_{i}, \mathrm{~T}\right) \gamma \mathrm{i}^{E}=f\left(\mathrm{y}_{i} \mathrm{~T}\right)
$$

5. Calcular las siguientes funciones que determinan un vector columna de dimensión $(\mathrm{r}+\mathrm{c})$
$\mathrm{F}(m)=\prod_{i=1}^{c}\left[\left(\gamma_{i} x_{i}\right)^{I}\right]^{\mathrm{v} i, m}-K_{m}(T) \quad \mathrm{m}=1, \ldots, \mathrm{r}$

$\mathrm{F}(n)=\left(\gamma_{n-r} x_{n-r)}\right)^{I}-\left(\gamma_{n-r} x_{n-r}\right)^{I I} \mathrm{n}=\mathrm{r}+1 \ldots, \mathrm{c}+\mathrm{r}$

6.Si $|\mathrm{F}|$ es mayor a la tolerancia establecida volver al paso dos para redefinir los valores de las variables. En este paso se utiliza un método como el de Mewton Raphson para ecuaciones algebraicas acopladas [16] empleando diferencias finitas para aproximar la derivada parcial.

7.Después de alcanzar el criterio de convergencia, los valores de $x, y$ y $\phi$ representan la solución del equilibrio líquido líquido reactivo. Hay que asegurarse que estos valores estén en el rango [0 1] para que la solución sea real.

En el presente trabajo se utiliza UMIQUAC y MRTL para el cálculo de los coeficientes de actividad y se trabaja con valores de la constante de equilibrio no dependientes de la temperatura, con el fin de simplificar los cálculos. Esto último es válido si se asume que el proceso de reacción transcurre prácticamente de manera isotérmica $\left(\Delta \mathrm{H}_{\mathrm{rxn}} \sim 0\right)$.

En un sistema con una sola reacción química, donde la suma de los coeficientes estequiométricos sea igual a cero, la ecuación (1) toma la forma:

$$
\mathrm{y}_{i}=\frac{\mathrm{Z}_{i}-\phi x_{i}+\left(\mathrm{v}_{i}^{T} / \mathrm{v}_{e}\right)\left((1-\phi) y_{e}+\phi x_{e}-z_{e}\right)}{1-\phi} \quad i=1 \ldots c-r
$$

\subsection{Representación del Equilibrio Simultáneo:}

El equilibrio simultáneo se representa mediante la conjugación del diagrama de equilibrio de fases y el de equilibrio de reacción y es llamado diagrama de ELLR o diagrama de fases con reacción química (para simplificar, en algunos casos, lo llamaremos simplemente diagrama de fases).

Cuando se trata de analizar una mezcla tricomponente la representación del equilibrio simultáneo se logra simplemente superponiendo 
el diagrama de ELL y el de Equilibrio de Reacción para definir los puntos de intersección. Para mezclas de cuatro o más componentes la representación gráfica requiere que estos equilibrios sean tratados de manera simultánea para generar la curva (en el caso de cuatro componentes) donde ambos se satisfacen.

\section{$\square$ Mezclas Ternarias:}

Se tomará como base los componentes hipotéticos planteados por Samant (1998) [10]. Se trata de un sistema que presenta la inmiscibilidad, pero la reacción y su respectiva constante de equilibrio son supuestas, al igual que el inerte. Todo esto se hace, debido a la dificultad de encontrar un sistema real ternario que se adapte a la configuración propuesta, es decir que A se descomponga sólo en $\mathrm{B}$ con $\mathrm{C}$ como inerte, $\mathrm{y}$ que a la vez $\mathrm{C}$ sea parcialmente miscible con A.

\begin{tabular}{|c|c|c|}
\hline \multirow{2}{*}{ COMPONENTES } & \multicolumn{2}{|c|}{ PARÁMETROS DE LA } \\
& ECUACIÓN UNIQUAC \\
\cline { 2 - 3 } & $\boldsymbol{r}$ & $\boldsymbol{q}$ \\
\hline (1) B & 2.204 & 2.072 \\
\hline (2) C & 0.920 & 1.400 \\
\hline (3) A & 2.870 & 2.410 \\
\hline
\end{tabular}

Parámetros de interacción Binaria (K)

\begin{tabular}{|l|l|l|}
\hline $\mathrm{a}(1,1)=0.0$ & $\mathrm{a}(1,2)=100.2$ & $\mathrm{a}(1,3)=-110.57$ \\
\hline $\mathrm{a}(2,1)=-85.939$ & $\mathrm{a}(2,2)=0.0$ & $\mathrm{a}(2,3)=546.80$ \\
\hline $\mathrm{a}(3,1)=267.8$ & $\mathrm{a}(3,2)=355.89$ & $\mathrm{a}(3,3)=0.0$ \\
\hline
\end{tabular}

Ejemplo 1: Considere un sistema compuesto por $A, B y C$ con la reacción $A \leftrightarrows B$, donde $C$ es un inerte parcialmente miscible con $A$.

La figura 1 es el diagrama de fases para este sistema, con constante de equilibrio $K=1.0$. La línea $\boldsymbol{a} \boldsymbol{P} \boldsymbol{b}$ es la línea binodal donde $\boldsymbol{P}$ es el punto de pliegue, y la curva $\boldsymbol{c} \boldsymbol{C}$ es la curva de reacción. Las líneas punteadas son líneas de liga y las flechas son líneas estequiométricas las cuales son la representación del avance de reacción para una mezcla inicial dada. Para este valor de $K$, las curvas de equilibrio de fases y de reacción no se intersectan, por lo que en equilibrio, el sistema está en una sola fase con una composición dada por el punto de intersección de la línea estequiométrica correspondiente a la mezcla inicial y la curva de equilibrio de reacción.

Para definir la(s) región(es) de dos fases, es decir, donde la mezcla en equilibrio se encuentra separada en dos fases líquidas, se dibujan las líneas estequiométricas que pasan por los puntos de corte de la curva de equilibrio de reacción (CER) y fásico (CEF) y la región comprendida entre ellas formará la región de dos fases. Por fuera de esta región el sistema siempre estará en una sola fase y la composición de equilibrio estará dada por la CER.

El diagrama 2 muestra el ejemplo anterior pero con una constante de equilibrio $\mathrm{Ka}=0.35$ con la cual sí hay intersección entre la CER y la CEF.

La unión de los puntos de corte de la CER y la CEF para cada región forma las Líneas de Liga Reactivas y estos dos puntos extremos ( $d y$ $e$, figura 2) son las composiciones de las fases en que se separa cualquier mezcla inicial que esté en la región de dos fases.

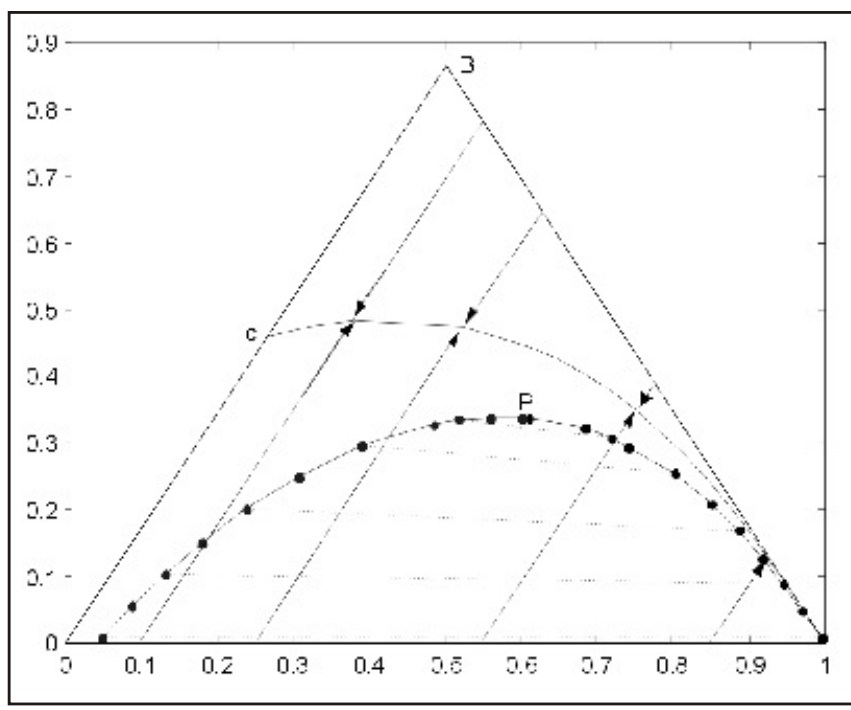

Figura 1. Diagrama de Fases para $\mathrm{A} \leftrightarrows \mathrm{B}$ con $\mathrm{K}=1.0$ en presencia del inerte $\mathrm{C}$

VOLUMEN 6 - No. 1 - Septiembre de 2004 


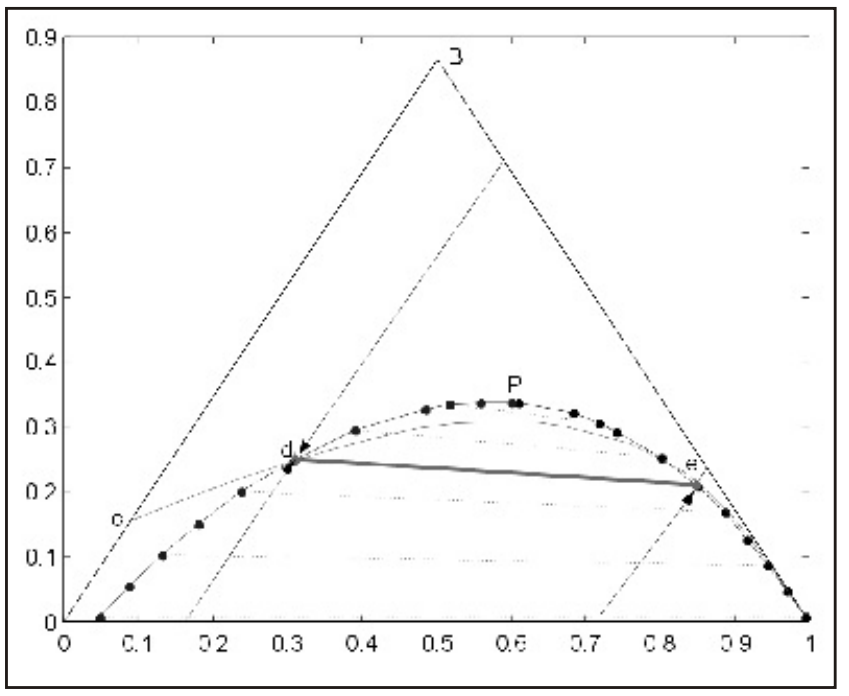

Figura 2. Diagrama de Fases para $\mathrm{A} \leftrightarrows \mathrm{B}$ con $\mathrm{K}=0.35$ en presencia del inerte $\mathrm{C}$

\section{Mezclas Cuaternarias:}

Los sistemas cuaternarios a presión y temperatura constante pueden representarse mediante un tetraedro con coordenadas de composición molar. La solución para el equilibrio fásico así como para el equilibrio de reacción serán superficies en este espacio. Si estas superficies no se intersectan el sistema siempre estará en una fase y la composición de equilibrio del sistema estará en la superficie de equilibrio de reacción (SER).

Si las superficies se intersectan, la región de intersección dará las composiciones de las fases en las que permanecerá el sistema en equilibrio. Bajo estas condiciones, a presión y temperatura constante, un sistema con una reacción tiene: 4(c)-2(f)-1(r)=1 grado de libertad, por lo que la región será una curva. En el resto del simplex concentracional el sistema en equilibrio tendrá una sola fase con composición perteneciente a la SER y dos grados de libertad. Cada región tiene un grado más que en el sistema tricomponente de la sección anterior, entonces, se puede emplear un Reactor Extractivo Multietapa
(REME) en contracorriente.

Ejemplo 2: El sistema de esterificación para la producción de Acetato de Butilo presenta miscibilidad parcial entre Acetato de Butilo Agua y Agua Butanol. Sigue la reacción: Ácido Acético $(A)+$ Butanol $(B) \stackrel{\leftrightarrows}{\rightarrow}$ Acetato de Butilo $(C)+\operatorname{Agua}(D)$. El butanol actúa también como solvente para la extracción.

El modelo de actividad empleado fue MRTL con los parámetros obtenidos por el simulador de procesos ASPEM PLUS ${ }^{\circledR} \quad 11.1$ [17]. La figura 3 representa el equilibrio líquido líquido (ELL), la figura 4 la superficie de equilibrio de reacción y la figura 5 el equilibrio líquido - líquido reactivo, todas a una temperatura de $293.15 \mathrm{~K}$. La constante de equilibrio de la reacción está dada en la referencia [11] como $\mathrm{K}_{\mathrm{eq}}=66.1$.

Tabla 2. Parámetros de la ecuación MRTL*

\begin{tabular}{|c|r|r|r|r|r|r|}
\hline Componente i & \multicolumn{1}{c|}{ B } & \multicolumn{1}{c|}{ D } & B & B & \multicolumn{1}{c|}{ A } & \multicolumn{1}{c|}{ A } \\
\hline Componente j & \multicolumn{1}{c|}{ D } & \multicolumn{1}{c|}{ C } & C & A & \multicolumn{1}{c|}{ D } & C \\
\hline aij & 204.2348 & \multicolumn{1}{c|}{168.1173} & 0 & 0 & -1.9763 & 0 \\
\hline aji & 90.5263 & 147.1602 & 0 & 0 & 3.3293 & 0 \\
\hline bij & -9291.7021 & -8343.6055 & 313.8322 & -381.5959 & 609.8886 & 17.3333 \\
\hline bji & -4983.1548 & -5855.2847 & -40.8184 & 550.1623 & -723.8881 & 261.2017 \\
\hline eij & -30.5804 & -23.5386 & 0 & 0 & 0 & 0 \\
\hline eji & -12.0592 & -22.1771 & 0 & 0 & 0 & 0 \\
\hline alfa & 0.2 & 0.2 & 0.3 & 0.3 & 0.3 & 0.3 \\
\hline
\end{tabular}

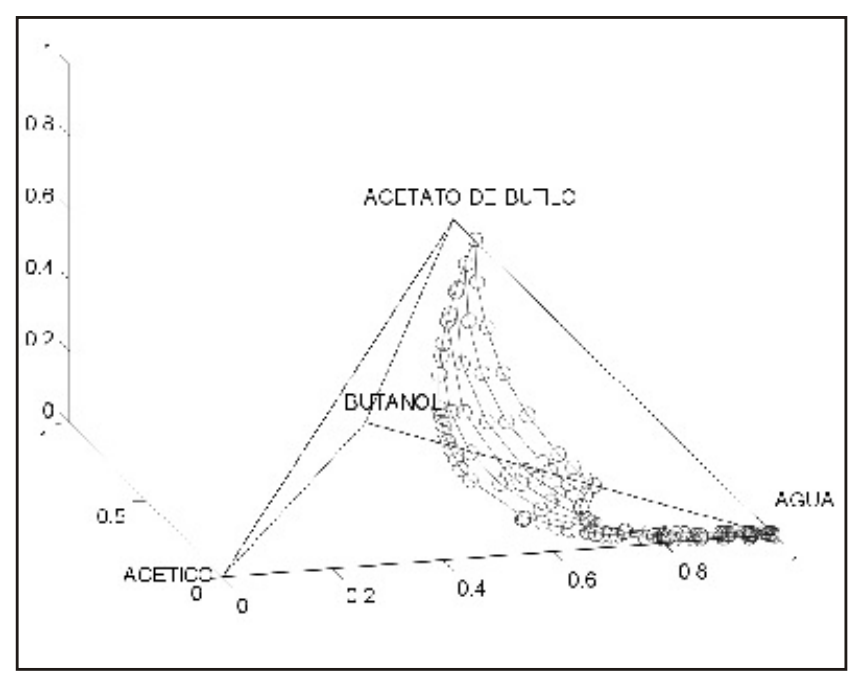

Figura 3. ELL. SISTEMA: Ácido Acético -Butanol - Acetato Butilio - Agua

$$
{ }^{*} \mathrm{~T}(\mathrm{~K}) \tau_{\mathrm{ij}}=\mathrm{a}_{\mathrm{ij}}+\mathrm{b}_{\mathrm{ij}} / \mathrm{T}+\mathrm{e}_{\mathrm{ij}} * \log (\mathrm{T})
$$




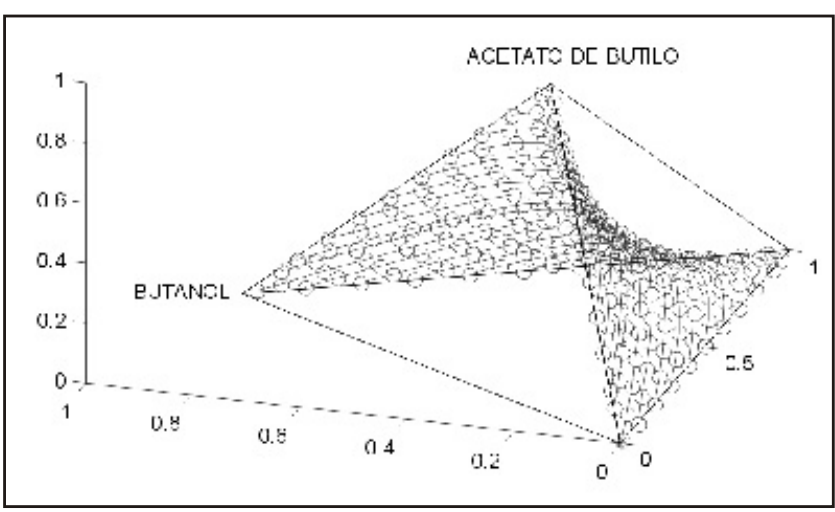

Figura 4. EQUILIBRIO QUÍMICO. SISTEMA Ácido Acético + Butanol $\leftrightarrows$ Acetato de Butilo + Agua

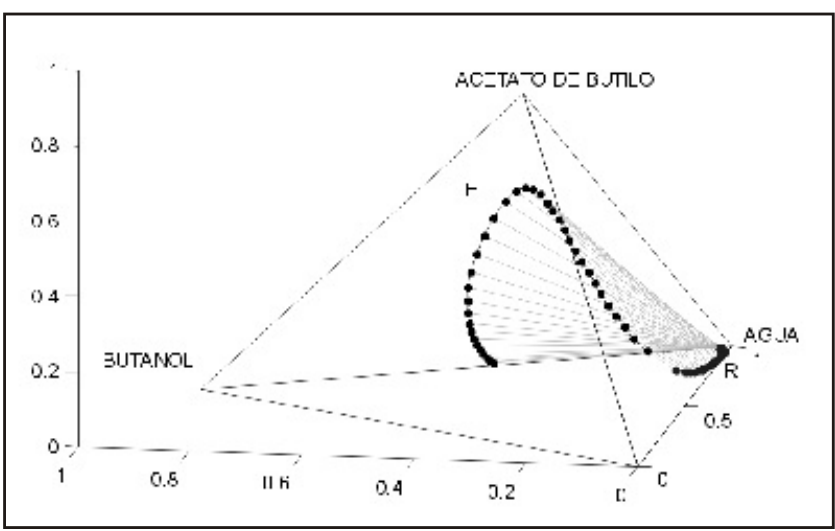

Figura 5. DIAGRAMA DE FASES. Sistema: Ácido Acético + Butanol $\leftrightarrows$ Acetato de Butilo + Agua

El equilibrio químico fue calculado y graficado con la ayuda de la ecuación (2). Según un análisis de la regla de fases, en equilibrio simultáneo para una mezcla de $C$ componentes con una sola reacción, las composiciones forman un subespacio de dimensión menor en el simplex concentracional. En nuestro caso entonces el equilibrio químico se representa por una superficie de tipo "silla" (ver Fig. 4). Cada punto de composición en ella corresponde a una afinidad química igual a cero, es decir la velocidad de reacción es también cero.

En el caso del equilibrio químico y físico simultáneos representado en la Fig. 5, los puntos extremos de las líneas indican las fases extracto y refinado en que se divide una mezcla inicial cuya composición esté en la región de dos fases.

Con la información obtenida para los ejemplos considerados, es entonces posible conocer las regiones del simplex concentracional donde el sistema en equilibrio se divide en dos fases líquidas y las composiciones de dichas fases.

Estas gráficas son fundamentales para 1) la comprensión de la trayectoria del proceso que permitirá continuar las investigaciones en el análisis de la estática aplicado a procesos de Reacción Extractiva; 2) en la construcción de un método geométrico partiendo de la representación del equilibrio simultáneo, para el diseño de Reactores Extractores Multietapa (REME).

\section{CONCLUSIONES}

Los procesos heterogéneos simultáneos logran una reconcentración de los reactivos, dando así la posibilidad de trabajar en la región cinética del simplexconcentracional.

El futuro de estos procesos a nivel económico y ambiental está en la minimización del consumo de energía, lo cual se beneficia conjugando la reacción con métodos indirectos como la extracción, que tiene gastos energéticos bajos comparados con procesos como destilación, cristalización, etc.

Se presenta un algoritmo para el cálculo del equilibrio líquido - líquido reactivo, ilustrado con dos ejemplos, a partir de los cuales se generaliza que, aunque los grados de libertad se vean reducidos en una unidad por la restricción que impone una reacción química en equilibrio, la solución del equilibrio simultáneo genera envolventes que siguen teniendo características similares a las encontradas en diagramas de equilibrio líquido líquido. 


\section{AGRADECIMIENTOS}

Dirección de investigaciones de la Universidad Macional de Colombia Sede Manizales por la financiación de la investigación.

\section{BIBLIOGRAFÍA}

[1] Pisarenko Yuri A., Serafimov L.A., Cardona C.A., Efremov D.L., Shuwalov A.S. Reactive Distillation Design: Analysis of the Process Statics. Reviews in Chemical Engineering. Freund Publishing House. Londres. Vol. 17. Mo.4.pp 253-327, 2001

[2] Cardona Carlos A., Pisarenko Yuri A. Análisis de Procesos Simultáneos Reacción Separación: Destilación Reactiva. En: II Taller Internacional De Análisis De Procesos. La Habana. Memorias. La Habana: ISPJAE (1999)

[3] Cardona, C. A. Aplicación de la Destilación Reactiva en Colombia para la Obtención de Acetatos y Aditivos Oxigenantes. Informe final, proyecto de investigación DIMA Universidad Macional de Colombia sede Manizales, febrero 2004.

[4] Pai Rajaram, Malone Michael, Doherty Michael. "Design of Reactive Extraction Systems for Bioproduct Recovery". AIChE J. 48, 3 514-526 (2002).

[5] Juang Ruey-Shin, Huang Ren-Hour. Equilibrium Studies on Reactive Extraction of Lactic Acid with an Amine Extractant. Chem. Eng. Journal. 65, 47-53 (1997).

[6] Bora M., Ghosh A., Dutta M., Mathur R., "Reactive Extraction of 6-Aminopenicillanic Acid with Aliquat-336: Equilibrium and Kinetics". Can. J. Chem. Eng.75, 520-526 (1997).

[7] Malinowski, Janusz. "Reactive Extraction for Downstream Separation of 1,3-Propanediol ". Biotechnol. Prog. 16, 76-79. (2000)

[8] Hatzinikolau D. y Wang $H$. "Extractive Fermentation Systems for Organic Acid Production" ". Can. J. Chem. Eng.70, 543552 (1992).

[9] Kollerup, Finn y Daugulis, Andrew. Ethanol Production by Extractive Fermentation Solvent Identification and Prototype
Development. Can. J. Chem. Eng. 64, 598606 (1986)

[10] Samant K. Y K.M. Mg. "Synthesis of Extractive Reaction Processes". AIChE J. 44, 13631381 (1998 a).

[11] Minotti M., Doherty M. y Malone M. "Design for simultaneous Reaction and Liquid- Liquid Extraction". Ind. Eng. Chem. Res. 37, 47484755. (1998)

[12] Serafimov, L.A., Zharov V.T., Timofeev, V.S., and Balashov, M.l., Rectification of multicomponent mixtures. II. Local and general characteristics of the trajectories of rectification processes at infinite reflux ratio, Acta Chim Academ Scientiar Hungar, 1973, 75(2), 193.

[13] Cardona Alzate, Carlos Ariel; Isabel Paz; Alejandro Agudelo, Mario. Vinyl acetate production by reactive distillation: analysis of feasible steady states based on the phase and chemical equilibrium. In: VI Conferencia Iberoamericana Sobre Equilibrio De Fases Para El Diseño De Procesos, 2002, Foz de Iguazu. Memorias de la VI Conferencia Iberoamericana sobre equilibrio de fases para el diseño de procesos. Rio de Janeiro: Epapers Services Editorials, 2002.

[14] Cardona Alzate, Carlos Ariel; Pisarenko, Yu.A.; Serafimov, Leonid A. Mew perspectives in C14 acetates production using reactive distillation: Acetic Acid or Acetic Anhydride as raw materials. En: 3rd European Congress of Chemical Engineering, 2001, MurembergGermany. Memories Frankfurt: ECCE.DECHEMA (CD version and abstracts in book), 2001

[15] Pisarenko, Yu., Serafimov, L., Cardona , C.A. Optimization of Reactive Distillation Processes. Theoretical Foundations Of Chemical Engineering, Moscow- Russia, v. 33, n. 5, p. 455-463, 1999.

[16]Rice, Richard; Do, Duong. Applied Mathematics and Modelling for Chemical Engineers. John Wiley \& Sons. 1995

[17] ASPEM PLUS Versión11.1. Aspen Technology, Inc. Estados Unidos. 2001 